\title{
Slow Passage Through Thresholds in Quantum Dot Lasers
}

\author{
E.A. Viktorov ${ }^{1,2,3}$, T. Erneux ${ }^{2}$, E.D.Kolykhalova ${ }^{4}$, V.V.Dudelev $^{5}$, J. Danckaert ${ }^{3}$, \\ K.K.Soboleva ${ }^{6}$, A.G.Deryagin ${ }^{5}$, I.I. Novikov ${ }^{5}$, M.V. Maximov ${ }^{5}$, A.E. Zhukov ${ }^{7}$, \\ V.M. Ustinov ${ }^{5}$, V.I.Kuchinskii ${ }^{5}$, W.Sibbett ${ }^{8}$, E.U.Rafailov ${ }^{9}$, G.S.Sokolovskii ${ }^{5}$ \\ 1-National Research University of Information Technologies, Mechanics and Optics, Saint Petersburg, Russia \\ 2-Optique Nonlinéaire Théorique, Campus Plaine, CP 231, 1050 Bruxelles, Belgium \\ 3-Applied Physics Research group (APHY), Vrije Universiteit Brussel, Pleinlaan 2, B-1050 Brussels, Belgium \\ 4-St.Petersburg State Electrotechnical University "LETI", St. Petersburg, Russia \\ 5-Ioffe Institute, St. Petersburg, Russia \\ 6-Peter the Great Saint Petersburg Polytechnical University, St. Petersburg, Russia \\ 7- Academic University, St. Petersburg, Russia \\ 8-University of St. Andrews, St. Andrews, UK and \\ 9-Aston Institute of Photonic Technologies, Aston University, Birmingham, UK
}

(Dated: August 28, 2016)

\begin{abstract}
A turn-on of a quantum dot (QD) semiconductor laser simultaneously operating at the ground (GS) and excited (ES) states is investigated both experimentally and theoretically. We find experimentally that the slow passage through the two successive laser thresholds may lead to significant delays in the GS and ES turn-ons. The difference between the turn-on times is measured as a function of the pump rate of change $\varepsilon$ and reveals no clear power law. This has motivated a detailed analysis of rate equations appropriate for two-state lasing QD lasers. We find that the effective time of the GS turn-on follows an $\varepsilon^{-1 / 2}$ power law provided that the rate of change is not too small. The effective time of the ES transition follows an $\varepsilon^{-1}$ power law but its first order correction in $\ln (\varepsilon)$ is numerically significant. The two turn-ons results from different physical mechanisms. The delay of the GS transition strongly depends on the slow growth of the dot population while the ES transition only depends on the time needed to leave a repellent steady state.
\end{abstract}

Semiconductor quantum dot (QD) based optical materials and devices have led to intensive research activities because of promising technological applications in biophotonics and optical communications. The recombination of ground state (GS) and excited state (ES) electrons and holes in QD lasers may contribute to simultaneous lasing at both states [1]. Simultaneous lasing has been investigated in steady-state operations [2-4], in dynamical regimes of feedback [5], saturable absorption [6, 7], and mode locking [8].

Turn-on experiments where the electrical pump is changed from a below to an above threshold provide important informations on the laser dynamical response. The turn-on time is defined as the time when either the GS or the ES intensity is quickly rising from nearly zero. The laser turn-on time depends on the rate of change of the pump as it has been theoretically predicted $[9,10]$ and experimentally confirmed [11]. The impact of the nonlinear and non-instantaneous capturing of the carriers into a dot on the laser turn-on dynamics has recently been investigated for QD lasers operating at the GS transition [13-15]. But the turn-on effects for QD lasers exhibiting both the GS and ES transitions remain currently unexplored.

In this paper, the QD laser turn-on is analyzed, experimentally and theoretically, when lasing occurs at both GS and ES transitions and the rise-time of the pump source is slow compared to the material and cavity time scales. Slow passages through single bifurcation points are well documented in nonlinear optics $[16,17]$ but slow passages through two successive thresholds are new to the best of our knowledge. Although the second transition is reminescent of a slow passage through a simple steady state bifurcation point, the first transition exhibits an unespected time history because some of the population variables need to be activated before the GS intensity may jump.

We experimentally find that the laser may turn-on with a significant $n s$ delay between the GS and ES emissions. Our main objective is to investigate theoretically the slow passage through both the GS and ES thresholds and determine the turn-on times as functions of the pump current rate of change. As we shall demonstrate, the passage through the GS bifurcation involves a two stage process that has a direct impact on the turn-on. On the other hand, the ES transition which comes after the GS transition depends on the time needed to reach the ES bifurcation point. Scaling laws are determined experimentally and analytically from rate equations for either small or large pump currents.

Experimentally, the studied QD laser structure was grown on a GaAs substrate by molecular-beam epitaxy. The active region included five layers of self-assembled InAs QDs separated with a GaAs spacer from a $5.3 \mathrm{~nm}$ thick covering layer of $I n_{0.14} G a_{0.86} A s$. Finally, the structure was processed into $4 \mu \mathrm{m}$-wide mesa stripe devices. The 1.5 to $2.5 \mathrm{~mm}$ long lasers with high- and antireflection coatings on the rear and front facets lase either at the GS (around $1265 \mathrm{~nm}$ ) or simultaneously at the GS and ES (around $1190 \mathrm{~nm}$ ) in the whole range of pumping. The laser did not show ground state quenching for increasing pump current. Short-pulsed electrical pump- 
ing was used to achieve high output power operation and avoid the effect of overheating on the output pulse shape.

Operation in the pulse-pumped regime is necessary to explore the turn-on dynamics. Pulses of $\sim 5$ ns risetime (measured at $10 \%-90 \%$ level) obtained from a high power (up to $2 A$ current) pulse source were used to turn the laser on. During the turn-on experiment, the intensity of the laser field first remains small until it quickly increases exponentially. The laser turn-on time defines the dynamical GS threshold. The laser output was detected using a high-speed pin detector with a cut-off frequency of $30 \mathrm{GHz}$ and a $50 \mathrm{GHz}$ digital oscilloscope. We simultaneously detect the total output and merely the ES output using a Bragg filter transmitting the shortwavelength and reflecting the long-wavelength radiation. We concentrate on the time difference between the rise-up of the GS output power and that of the ES as measured by the photodetector. Further details of the experimental technique can be found in [14].

Typical experimental time traces are shown in Fig. 1. The pump current increased linearly in time as $J^{\prime}\left(t^{\prime}\right)=\left(J_{p} / \tau_{r}\right) t^{\prime}$ where $\tau_{r}=5 n s$ is a fixed rise time and $J_{p}$ is the maximum pump current. Changing $J_{p}$ allows us to change the sweeping rate and to analyze its effect on the two successive turn-ons. The laser turn-on at the GS wavelength is seen by an exponential increase of the output power. After the laser turns on, the GS output quickly relaxes to a slowly varying steady state that follows the increasing pump current. The ES output remains OFF about $2 n s$ after the GS turn-on. We then observe the ES turn-on as a new exponential increase of the ES output power. It is followed by a relaxation to a slowly varying steady state where both GS and ES intensities are non zero. The reservoir charge carrier lifetime is typically of the order of $0.1 \mathrm{~ns}$ and cannot explain the $n s$ time difference between the GS and ES transitions. Both turn-ons experience significant delays after passing the GS and ES static bifurcation points due to the inertia of the system response. These delays result from different physical mechanisms. The delay of the GS bifurcation is controlled by the slow growth of the dot population. The delay of the ES bifurcation results from the time needed to leave a repellent steady state. Fig. 1b shows numerical simulations of the dimensionless rate equations (1)-(4). The intensities are represented in terms of the original time $t^{\prime}=t \times \tau_{\text {phot }}$ where $\tau_{\text {phot }}=10$ ps. All other parameters are documented in the figure caption.

The time difference $\Delta t$ between the GS and ES turnon times shown in Fig.1a depends on the rate of change of the pump current which is increased by increasing $J_{p}$. The functional dependence of $\Delta t$ on $\mathrm{J}_{p}$ is examined by fitting the experimental data (see Fig. 2). The fits suggest different scaling laws for the low and high values of $J_{p}$. However, we need to be cautious in interpreting the power law fits. In particular, we have noted that fitting the data for the high pump currents leads to quite different answers depending on the number of data we consider. This motivates the analysis of the laser rate
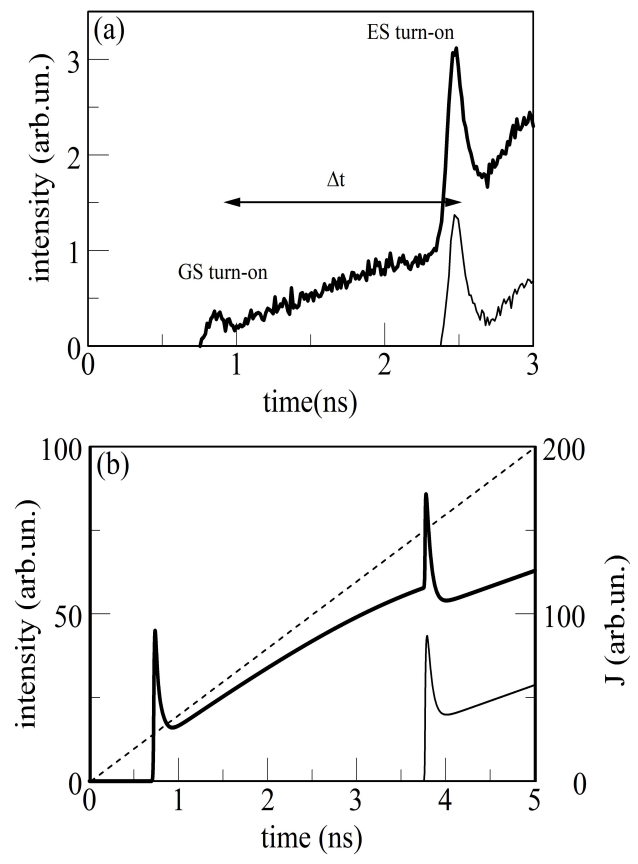

FIG. 1: Total (GS + ES) and filtered (ES) intensities. (a) Experimental turn-on traces for $J_{p}=1 A$ and rise time $\tau_{r}=5$ ns. (b) Numerical simulations using Eqs. (1 )-(4). The values of the fixed parameters are $g=2, B_{g}^{c a p}=10, B_{e}^{c a p}=100, \eta=$ $0.01 . J(t)=0.4 t(0<t<500)$. All intensities are represented in terms of $t^{\prime}=\tau_{\text {phot }} t$ where $\tau_{\text {phot }}=10 \mathrm{ps}$. The dashed line in Fig. 1(b) shows the pump current $J$ as a function of the orginal time $t^{\prime}$. $\Delta t$ denotes the difference between the GS and ES turn-on times. The thick (thin) lines correspond to the total GS + ES (ES) laser output.

equations by looking for approximations of the effective turn-on times $\left(t_{t h 1}\right.$ and $\left.t_{t h 2}\right)$.

The complexities of intradot dynamics constitute multiple challenging issues to the modeling of simultaneous lasing in QD lasers. Whereas considering different physical impacts on simultaneous lasing, all the models [1-3] consider the appearance of simultaneous lasing as the result of steady bifurcation transitions. In addition to the laser $\mathrm{OFF}$ state, there exist three laser ON steady states, namely (1), GS (ES) - ON (OFF), (2) ES (GS) - ON (OFF), and (3) simultaneous lasing with both GS and ES states ON. In our set-up, the laser first undergoes a bifurcation from the OFF state to the GS (ES) - ON (OFF) state as the pump parameter is slowly increased. It then passes through a second bifurcation point where simultaneous lasing in the GS and ES state is possible. Because the pump parameter is changing in time, the actual bifurcation transitions do not occur at the static bifurcation points but appear later. The effects of slow passages through bifurcation points need to be carefully analyzed for each case [16]. Here, we are facing the problem of two successive steady bifurcations where the GS and ES states sequentially become active.

We are using a rate equation model that largely corresponds to the excitonic model which were originally pro- 


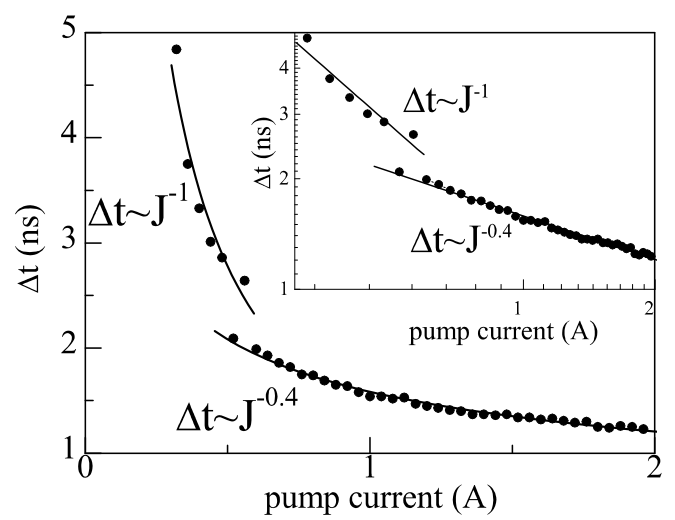

FIG. 2: Experimentally measured $\Delta t$ at room temperature $\left(20^{\circ} C\right)$ and fitting curves. We identify different behaviors for low $\Delta t \sim J^{-1}$ and high $\Delta t \sim J^{-0.4}$ pump currents $J$.

posed in $[1,18]$ and accounts for the essential intradot processes. The effect of gain compression is extremely weak in the vicinity of the thresholds and is, therefore, neglected. The model consists of the following equations for the GS (ES) electric field intensity $I_{g}\left(I_{e}\right)$, the occupational probabilities of the GS (ES) in a $\operatorname{dot} \rho_{g}\left(\rho_{e}\right)$, and the carrier density $n$ in the wetting layers $(\mathrm{WL})$, scaled to the $2 \mathrm{D} \mathrm{QD}$ density per layer. They are given by

$$
\begin{aligned}
I_{g, e}^{\prime} & =\left[g_{g, e}\left(2 \rho_{g, e}-1\right)-1\right] I_{g, e}+\delta, \\
\rho_{g}^{\prime} & =\eta\left[2 F_{g}-\rho_{g}-g_{g}\left(2 \rho_{g}-1\right) I_{g}\right], \\
\rho_{e}^{\prime} & =\eta\left[F_{e}-F_{g}-\rho_{e}-g_{e}\left(2 \rho_{e}-1\right) I_{e}\right], \\
n^{\prime} & =\eta\left[J(t)-n-4 F_{e}\right] .
\end{aligned}
$$

Prime means differentiation with respect to $t \equiv t^{\prime} / \tau_{\text {phot }}$ where $\tau_{\text {phot }}=10 p s$ is the photon lifetime. $\eta \equiv$ $\tau_{\text {phot }} \tau^{-1}<<1$, where $\tau$ denotes the carrier recombination time. The gain $g_{g, e}\left(2 \rho_{g, e}-1\right)$ is defined by the dot population and a $g_{g, e}$-factor, where $g_{g}=2 g$ and $g_{e}=4 g$. The factors 2 and 4 account for the spin degeneracy in the quantum dot energy levels. We define $g$ as the effective gain factor scaled to the cavity losses, and assume the gain factors and the cavity losses to be identical for both GS and ES. $F_{g} \equiv B_{g}^{c a p} \rho_{e}\left(1-\rho_{g}\right)-B_{g}^{e s c} \rho_{g}\left(1-\rho_{e}\right)$ and $F_{e} \equiv B_{e}^{c a p} n\left(1-\rho_{e}\right)-B_{e}^{e s c} \rho_{e}$,where the terms $1-\rho_{g, e}$ correspond to Pauli blocking. The time-dependent recovery of the QD gain is described by $B_{g}^{c a p} \equiv \tau / \tau_{g}^{c a p}$ and $B_{e}^{c a p} \equiv \tau / \tau_{e}^{c a p}$, where $\tau_{g}^{c a p}$ and $\tau_{e n}^{c a p}$ denote capture times. The dimensionless coefficients $B_{g, e}^{c a p}$ are in the 10 to 100 range. To determine the escape rates $B_{g, e}^{e s c}$, we use Kramer relation [19] linking the capture $B_{g, e}^{c a p}$ and the escape $B_{g, e}^{e s c}$ rates as

$$
B_{g, e}^{e s c}=B_{g, e}^{c a p} \exp \left(-\Delta E_{g, e} / k_{B} T\right),
$$

where $k_{B}$ is the Boltzmann constant and $T$ is the plasma temperature. We assume the GS and ES spacing as $\Delta E_{g} \simeq 50 \mathrm{meV}$ and $\mathrm{ES}$ and WL spacing as $\Delta E_{e} \simeq 150$

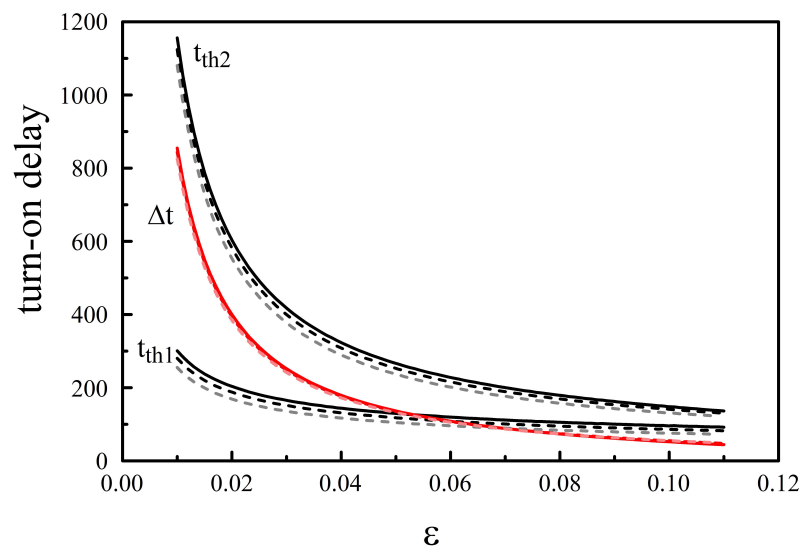

FIG. 3: Bifurcation transition times $t_{t h 1}$ and $t_{t h 2}$ and $\Delta t=$ $t_{t h 2}-t_{t h 1}$ as functions of $\varepsilon=J_{p} \tau_{p h} / \tau_{r}(0.01<\varepsilon<0.1)$ in the units of $\tau_{p h}$. The values of the fixed parameters are $J_{0}=0.1$, $g=2, B_{g}^{\text {cap }}=10, B_{e}^{\text {cap }}=100, B_{g, e}^{\text {esc }}=0$, and $\delta=10^{-12}$ (full lines). The two broken lines, from bottom to top are for $\delta=10^{-3}$ and $\delta=10^{-6}$, respectively. The intial conditions corresponds to GS-OFFstate at $J=J_{0}$.

meV. At room temperature $k_{B} T=25 \mathrm{meV}$. In Eq. (4), $J(t)=J_{0}+\varepsilon t$ is the time-dependent pump current where $\varepsilon \equiv J_{p} \tau_{\text {phot }} / \tau_{r}$ is the rate of change and $J_{0}<<1$ is the initial value of the pump current. A small parameter $\delta$ in the right hand side of Eq. (1) mimics the effect of noise that prevent the intensities to approach extremely small values during the slow increase of $J(t)$.

We wish to analyze the slow passage through the two successive bifurcation points in detail. To this end, we neglect the escape processes $\left(B_{g, e}^{e s c}=0\right)$ and plan to determine the turn-on times of first $I_{g}$ and then $I_{e}$ as a function of $\varepsilon$. The steady bifurcation points are located at $J_{G}$ and $J_{E}$ and the actual turn-ons occur at $J_{t h 1}>J_{G}$ and $J_{t h 2}>J_{E}$, respectively. From the steady state equations, we determine $J_{G}$ and $J_{E}$ analytically. If $B_{e, g}^{c a p}>10$, they are well approximated by the following expressions

$$
J_{G}=\left(1+\frac{1}{2 g}\right) \text { and } J_{E}=\left(1+\frac{1}{4 g}\right) B_{g}^{c a p}\left(1-\frac{1}{2 g}\right) .
$$

The expressions in (6) indicate that $J_{G}$ is independent of the $B_{e, g}^{c a p}$ while $J_{E}$ is proportional to $B_{g}^{c a p}$. In Fig. 3, we show the numerically computed turn-on times $t_{t h 1}$, $t_{t h 2}$, and $\Delta t=t_{t h 2}-t_{h 1}$ as functions of $\varepsilon$. The times $t_{t h 1}$ and $t_{t h 2}$ are determined numerically as the times where $I_{g, e}(t)>I_{\text {thres }}$. The threshold value $\mathrm{I}_{\text {thres }}=8 \times 10^{-3}$ is chosen arbitrarily and is not significant because the exponential growths of $I_{g}$ and $I_{e}$ are quasi instantaneous at turn-on. The broken lines corresponds to higher values of the noise parameter $\delta$. As we may expect, the bifurcation transitions appear sooner if $\delta$ is increased although its effect is not significant.

Fitting $\Delta t$ as a power law $y=a x^{b}$ gives an exponent $b=-1.24$ which doesn't suggest a classical scaling law such as -1 or $-1 / 2$ [16]. On the other hand, fitting $t_{t h 1}$ and $t_{t h 2}$ as power laws is more interesting. For $t_{t h 1}$, we 


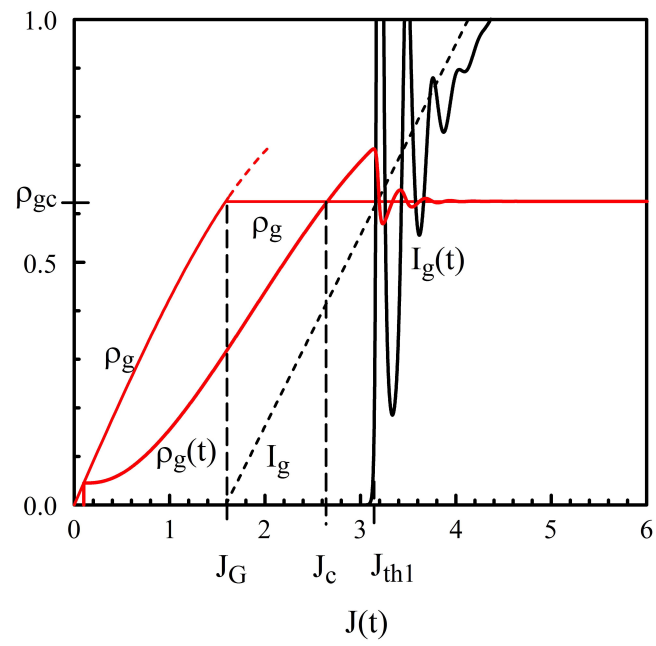

FIG. 4: Blow up of the GS transition. The broken lines labeled by $\rho_{g}$ and $I_{g}$ represent the steady states as function of $J(\varepsilon=\delta=0) . \quad J=J_{G}, J_{c}$, and $J_{t h 1}$ denote the GS steady bifurcation point, the point where $\rho_{g}>\rho_{g c}=0.625$ for the first time, and the point where $I_{g}(t)$ starts to grow, respectively. The fixed parameters are the same as in Fig. 3 with $\varepsilon=0.01$ and $\delta=10^{-12}$. The slow passage starts from the GS-OFF state at $J=J_{0}$.

find $b=-0.48$ and for $t_{t h 2}$, we obtain $b=-0.88$. The first threshold exhibits a clear $\varepsilon^{-1 / 2}$ scaling law. The second threshold suggests an $\varepsilon^{-1}$ scaling law but only qualitatively. We conclude that $\Delta t=t_{t h 2}-t_{t h 1}$ cannot be fitted by a simple scaling law and that the two turn-ons are controlled by different physical mechanisms. Fig. 4 shows that the GS intensity turn-on is a two stage process. We recall that $J=J_{G}$ is the laser stability threshold in the absence of the slow variation of the pump $(\varepsilon=0)$ while $J_{t h 1}$ corresponds to the real change of stability of the GSOFF steady state if $\varepsilon \neq 0$. We define the first stage as the trip from $J_{G}$ to $J=J_{c}(t)$. $J_{c}(t)$ marks the p oint where $\rho_{g}(t)$ surpasses $\rho_{g c} \equiv 1 / 2+1 /(4 g)$, which, according to Eq. (1) for $I_{g}$ with $g_{g}=2 g$, marks the point where the gain $g_{g}\left(2 \rho_{g}-1\right)-1$ becomes positive. $J_{c}(t)$ depends on the temporal evolution of $\rho_{g}(t)$ which considerably deviates from its steady state value $\rho_{g}$ if $\varepsilon=0$. As shown below, this deviation is $O(1)$ if $\varepsilon / \eta=O(1)$ and will be even more dramatic if $\varepsilon / \eta>>1$. The second stage of the GS turn-on corresponds to the trip from $J_{c}(t)$ to $J_{t h 1}$.

In order to analyze the first stage where $I_{g}$ remains close to zero, we note from the numerical solution that $\rho_{e}=O\left(\left(B_{g}^{c a p}\right)^{-1}\right)$ and $n=O\left(\left(B_{e}^{c a p}\right)^{-1}\right)$. These scalings motivate introducing $x=B_{e}^{c a p} n$ and $y=B_{g}^{c a p} \rho_{e}$. Neglecting all $\left(B_{g, e}^{c a p}\right)^{-1}$ small terms,Eqs. (2)-(4) simplify as

$$
\begin{aligned}
\rho_{g}^{\prime} & =\eta\left[2 y\left(1-\rho_{g}\right)-\rho_{g}-g_{g}\left(2 \rho_{g}-1\right) I_{g}\right] \\
y^{\prime} & =\eta B_{g}^{c a p}\left[x-y\left(1-\rho_{g}\right)\right] \\
x^{\prime} & =\eta B_{e}^{c a p}(J-4 x) .
\end{aligned}
$$

From the coefficients multiplying the right hand sides of these equations, we note that $x$ and $y$ are fast variables compared to $\rho_{g}$ and quickly approach their quasi-steady state values given by

$$
\begin{aligned}
& x=J / 4, \\
& y=J /\left(4\left(1-\rho_{g}\right)\right) .
\end{aligned}
$$

The remaining Eq. (7) can be rewritten as

$$
\rho_{g}^{\prime}=\eta\left(J / 2-\rho_{g}-g_{g}\left(2 \rho_{g}-1\right) I_{g}\right) .
$$

Before $I_{g}$ quickly turns on, $I_{g}$ remains close to zero. Neglecting the term multiplying $I_{g}$ in Eq. (12), and with the initial condition $\rho_{g}(0)=J_{0} / 2$, we determine the following solution for $\rho_{g}$

$$
\rho_{g}(t)=\frac{J(t)}{2}-\frac{\varepsilon}{2 \eta}(1-\exp (-\eta t)) .
$$

The second term in (13) indicates an important effect of the slowly varying pump since $\rho_{g}(t)$ substantially deviates from its quasi-steady regime, $\rho_{g}=J(t) / 2$, if $\varepsilon / \eta=O(1)$ or larger. Having $\rho_{g}(t)$, we solve Eq.(1) for $I_{g}$ which is separable. We find

$$
I_{g}=I_{g}(0) \exp \left[\eta^{-1} g_{g} F(\eta t)\right]
$$

where the growth rate $F(s)$ is defined by

$$
F(s) \equiv \frac{\varepsilon}{\eta}(1-\exp (-s))+\left[J_{0}-1-g_{g}^{-1}-\frac{\varepsilon}{\eta}\right] s+\frac{\varepsilon}{\eta} \frac{s^{2}}{2} .
$$

The function $F(s)$ is negative during the interval $0<s<$ $s_{t h 1} \equiv \eta t_{t h 1}$ which means that $I_{g}$ is exponentially small during this time interval. On the other hand, $I_{g}$ becomes exponentially large as soon as $s>s_{t h 1}$. The critical time $s_{t h 1}$ is defined as the non-zero root of Eq.(15). In implicit form, $s_{t h 1}=s_{t h 1}(\varepsilon / \eta)$ is given by

$$
\frac{\varepsilon}{\eta}=\frac{\left(1-J_{0}+g_{g}^{-1}\right) s_{t h 1}}{1-\exp \left(-s_{t h 1}\right)-s_{t h 1}+\frac{s_{t h 1}^{2}}{2}} .
$$

In Fig 5 , we compare $t_{t h 1}=s_{t h 1} / \eta$ where $s_{t h 1}$ is provided by Eq. (16) with the numerical estimate previously shown in Fig. 3 . The agreement is quantitative for $5 \times 10^{-2}<\varepsilon<1.1 \times 10^{-1}$. If $\varepsilon<5 \times 10^{-2}$, the analytical solution overestimates the turn on delay found numerically. It is worthwhile to look for the $\varepsilon / \eta$ large limit of (16) which is also shown in Fig. 5. In this limit, $s_{t h 1} \rightarrow 0$ as

$$
s_{t h 1}=\sqrt{6\left(1-J_{0}+g_{g}^{-1}\right) \frac{\eta}{\varepsilon}}
$$

which implies an $\varepsilon^{-1 / 2}$ scaling law for $t_{t h 1}=s_{t h 1} / \eta$.

In summary, the time $t_{t h 1}$ of the GS bifurcation transition follows an $\varepsilon^{-1 / 2}$, law in first approximation, and the delay is significant as soon as $\varepsilon / \eta$ is $O(1)$ or larger.

We next concentrate on how $I_{e}$ turns on. $I_{e}$ remains close to zero until $J=J_{t h 2}$. Fig. 6 shows the delayed 


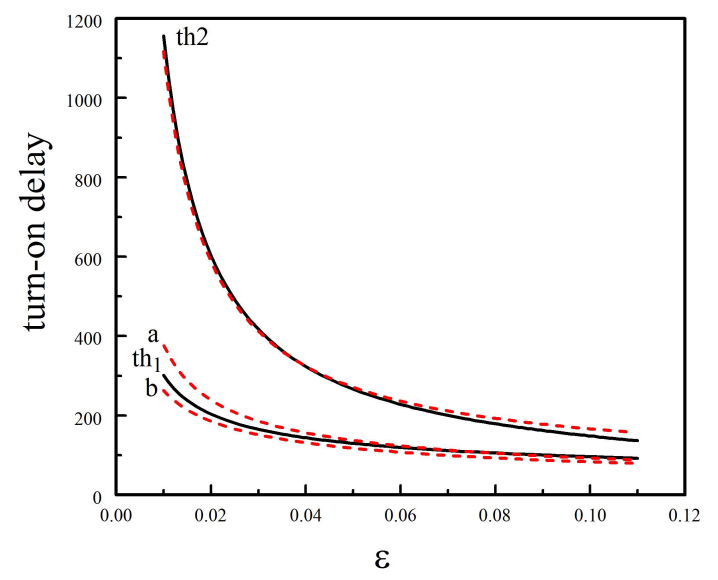

FIG. 5: Comparison between analytical and numerical estimates of the turn-on delays. Same values of the parameters as in Fig. 3 with $\delta=10^{-12}$ and $\eta=0.01$. Curves labeled by $a$ and $b$ are drawn from Eq. (16) and (17), respectively.

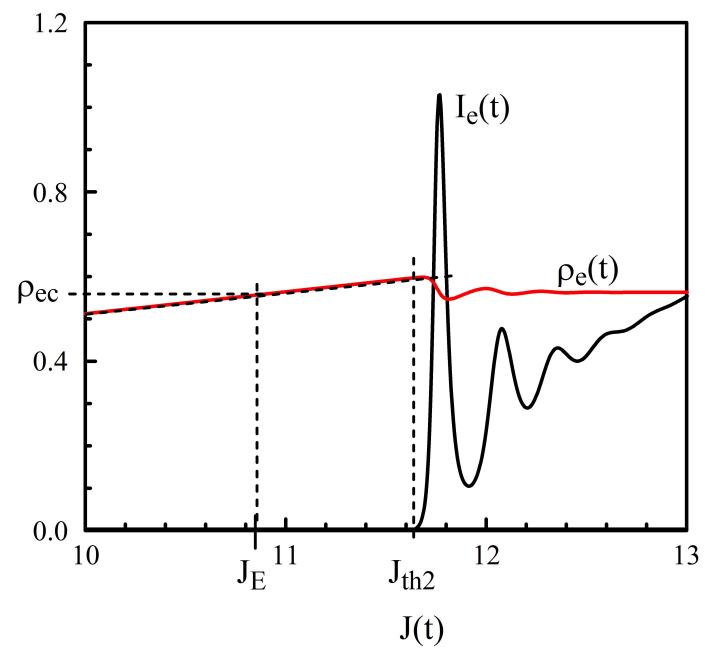

FIG. 6: Blow up of the ES transition. $J_{E}$ and $J_{t h 2}$ denote the ES bifurcation point and the point where $I_{e}$ starts to grow. respectively. The fixed parameters are the same as in Fig. 3 with $\varepsilon=0.01$ and $\delta=10^{-12}$. The slow passage started from the GS-OFF state at $J=J_{0}$.

bifurcation transition as $J(t)$ passes the ES static bifurcation point $J_{E}$. Close to the ES bifurcation point, $I_{g}, \rho_{g}$, $\rho_{e}$, and $n$ are following the GS steady state. From the expressions of the GS steady state, we find that

$$
\rho_{e}=\frac{J}{4\left[B_{g}^{c a p}\left(\frac{1}{2}-\frac{1}{4 g}\right)+1\right]}
$$

where we took into account that $B_{e}^{c a p}$ is large. Eq. (1) for $I_{e}$ can then be rewritten as

$$
I_{e}^{\prime}=\alpha\left(J-J_{E}\right) I_{e}+\delta
$$

where $\alpha$ and $J_{E}$ are defined by

$$
\begin{aligned}
\alpha & =\frac{4 g}{2\left[B_{g}^{c a p}\left(\frac{1}{2}-\frac{1}{4 g}\right)+1\right]}, \\
J_{E} & =2\left[B_{g}^{c a p}\left(\frac{1}{2}-\frac{1}{4 g}\right)+1\right]\left(1+\frac{1}{4 g}\right) .
\end{aligned}
$$

Eq. (19) is linear and can be solved with the initial condition $I(0)=I_{0}$. The solution is given by

$$
\begin{aligned}
I= & I_{0} \exp \left[\frac{\alpha}{2 \varepsilon}\left[\left(\varepsilon t+\left(J_{0}-J_{E}\right)\right)^{2}-\left(J_{0}-J_{E}\right)^{2}\right]\right] \\
& +\delta \sqrt{\frac{2}{\alpha \varepsilon}} \exp \left[\frac{\alpha}{2 \varepsilon}\left[\left(\varepsilon t+\left(J_{0}-J_{E}\right)\right)^{2}\right]\right] \\
& \times \frac{\sqrt{\pi}}{2}\left\{\begin{array}{c}
\operatorname{erf}\left[\sqrt{\frac{\alpha}{2 \varepsilon}}\left(\varepsilon t+J_{0}-J_{E}\right)\right] \\
-\operatorname{erf}\left[\sqrt{\frac{\alpha}{2 \varepsilon}}\left(J_{0}-J_{E}\right)\right]
\end{array}\right\}
\end{aligned}
$$

where $y=\operatorname{erf}(x)$ is the error function. Assuming now $J(t)-J_{E}$ as a positive $O(1)$ quantity and noting that $J_{0}-J_{E}$ is a negative $O(1)$ quantity, $\operatorname{erf}( \pm \infty)= \pm 1$ as $\varepsilon \rightarrow 0$. The expression (22) then reduces to a sum of two exponentials given by

$$
\begin{aligned}
I= & I_{0} \exp \left[\frac{\alpha}{2 \varepsilon}\left[\left(\varepsilon t+\left(J_{0}-J_{E}\right)\right)^{2}-\left(J_{0}-J_{E}\right)^{2}\right]\right] \\
& +\delta \sqrt{\frac{2 \pi}{\alpha \varepsilon}} \exp \left[\frac{\alpha}{2 \varepsilon}\left[\left(\varepsilon t+\left(J_{0}-J_{E}\right)\right)^{2}\right]\right]
\end{aligned}
$$

The first exponential is an $O(\exp (-1 / \varepsilon))$ small quantity for $0<t<-2 \varepsilon^{-1}\left(J_{0}-J_{E}\right)$. On the other hand, the second exponential is an $O(\exp (1 / \varepsilon))$ large quantity as soon as $J$ passes $J_{E}$. However, its action will be delayed if we assume $\delta$ as an $O(\exp (-1 / \varepsilon))$ small quantity. The critical time above which the second exponential will increase is then given by

$$
t_{t h 2}=\frac{J_{E}-J_{0}}{\varepsilon}-\frac{2}{\alpha}\left[\ln \left(\delta \sqrt{\frac{2 \pi}{\alpha}}\right)-\frac{1}{2} \ln (\varepsilon)\right] .
$$

Moreover, the value of $J$ at $t=t_{t h 2}$ is

$$
J_{t h 2}=J_{E}-\frac{2 \varepsilon}{\alpha}\left[\ln \left(\delta \sqrt{\frac{2 \pi}{\alpha}}\right)-\frac{1}{2} \ln (\varepsilon)\right] .
$$

We have verified that the approximation (25) is in quantitative agreement with the value obtained by integrating Eq. (19) numerically (the fixed parameters are the same as in Fig. 3, $\varepsilon=0.01$ and $\delta=10^{-12}$ ). The first term in (24) suggests an $\varepsilon^{-1}$ scaling law but the second term in (24) indicates a correction in $\ln (\varepsilon)$ which can be significant.

We reported on the turn-on dynamics of a QD laser operating simultaneously at the GS and ES which can be important for applications such as all-optical switches, 
and converters used in all-optical networks. The slow change of the pump current is responsible for delayed bifurcations and the time interval between the GS and ES effective transitions is recorded as a function of the rate of change $\varepsilon$. Fitting the data by a simple power law is however delicate. By studying the GS and ES slow passage problems numerically and analytically, we found that the GS transition is delayed by an $\varepsilon^{-1 / 2}$ quantity which becomes significant if the ratio $\varepsilon / \eta$ is $O(1)$. On the other hand, the slow passage through the ES transition is characterized by a $\varepsilon^{-1}$ scaling law smoothed by a $\ln (\varepsilon)$ correction term. As illustrated in Fig. 3, the main contribution to $\Delta t$ comes from the ES transition time for small rates of changes and we may then expect an $\varepsilon^{-1}$ power law dependence. On the hand, for larger values of the rate of change, the two turn-on times are com- ing closer and $\Delta t$ is the difference between two different scaling laws.

\section{Acknowledgments}

This work was partially funded by the Erasmus Mundus External Cooperation Window (EMECW) programme under Project No. 141085-EM-1-2008-BEERAMUNDUS-ECW-L02, the Research Foundation Flanders (FWO), the Fonds National de la Recherche Scientifique and the Belgian Science Policy Office under Grant No. IAP-7/35 "photonics@be." Support by the Russian Ministry of Science and Education (Grant ID: RFMEFI60714X0101) is acknowledged.
[1] A. Markus, J. X. Chen, C. Paranthoen, A. Fiore, C. Platz, and O. Gauthier-Lafaye, Appl. Phys. Lett. 82, 1818(2003).

[2] E.A.Viktorov, P. Mandel,Y. Tanguy, J. Houlihan, and G. Huyet, Appl. Phys. Lett. 87, 053113(2005).

[3] M. Gioannini, J. Appl. Phys. 111, 043108(2012).

[4] M. Abusaa, J. Danckaert, E. A. Viktorov, and T. Erneux Phys. Rev. A 87, 063827 (2013).

[5] E. A. Viktorov, P. Mandel, I. O'Driscoll, O. Carroll, G. Huyet, J. Houlihan, and Y. Tanguy, Opt. Lett. 31, 2302 (2006).

[6] E. A. Viktorov, M. A. Cataluna, L. O'Faolain, T. F. Krauss, W. Sibbett, E. U. Rafailov, and P. Mandel, Appl. Phys. Lett. 90, 121113 (2007).

[7] A. Markus, M. Rossetti, V. Calligari, D. Chek-Al-Kar, J. X. Chen, A. Fiore, and R. Scollo, J. Appl. Phys. 100, 113104 (2006).

[8] M. A. Cataluna, W. Sibbett, D. A. Livshits, J. Weimert, A. R. Kovsh, and E. U. Rafailov, Appl. Phys. Lett. 89, 081124 (2006).

[9] P. Mandel, T. Erneux, Phys. Rev. Lett. 53, 1818 (1984).

[10] T. Erneux, P. Mandel, Phys. Rev. A 39, 5179 (1989).

[11] J. I. Alexander, K. V. Vasavada, G. Vemurii, Quantum Semiclass. Opt. 7, 467 (1995).
[12] V. Booth, T.W. Carr, T. Erneux, SIAM J. Appl. Math., 57, 1406 (1997).

[13] K. Lüdge and E. Schöll, IEEE J. Quantum Electron. 45, 1396 (2009).

[14] G. S. Sokolovskii, V. V. Dudelev, E. D. Kolykhalova, A. G. Deryagin, M. V. Maximov, A. M. Nadtochiy, V. I. Kuchinskii, S. S. Mikhrin, D. A. Livshits, E. A. Viktorov and T. Erneux, Appl. Phys. Lett. 100 , 081109 (2012).

[15] G. S. Sokolovskii, V. V. Dudelev, E. D. Kolykhalova, A. G. Deryagin, M. V. Maximov, A. M. Nadtochiy, V. I. Kuchinskii, S. S. Mikhrin, D. A. Livshits, E. A. Viktorov and T. Erneux, J. of Physics: Conference Series 461, 012030, (2013).

[16] T. Erneux and P. Glorieux, Laser Dynamics, Camb. Univ. Press, Cambridge U.K. (2010).

[17] M. C. Torrent and M. San Miguel, Phys. Rev. A 38, 245 (1988).

[18] A. Markus, J. X. Chen, O. Gauthier-Lafaye, J.-G. Provost, C. Paranthoen, and A. Fiore, IEEE J. Sel. Top. Quantum Electron. 9, 1308 (2003).

[19] L. V. Asryan and R. A. Suris, Semicond. Sci. Technol. 11, 554 (1996). 